\title{
INTER-RELAÇÃO OU COEXISTÊNCIA: POSSÍVEIS (DES)ARTICULAÇÕES ENTRE A FIBROMIALGIA E A DEPRESSÃO
}

\section{INTER-RELACIÓN O COEXISTENCIA: POSIBLE (DES) ARTICULACIÓN ENTRE FIBROMIALGIA Y DEPRESIÓN}

\section{INTERRELATIONSHIP OR COEXISTENCE: POSSIBLE (DIS) ARTICULATIONS BETWEEN FIBROMYALGY AND DEPRESSION}

\author{
Karoliny Paula da Silva ${ }^{1}$; Higor Bezerra Lima²; André Cabral ${ }^{3}$
}

DOI: $\underline{\text { https://doi.org/10.31692/978-65-991061-9-4.1-13 }}$

\begin{abstract}
RESUMO
O escopo deste artigo é analisar como é estabelecida a relação entre a Síndrome de Fibromialgia e a depressão, vista a alta demanda de pacientes acometidos pelas enfermidades. Para nos fundamentar teoricamente, partimos da concepção de Mello-Filho et al (2010) sobre fibromialgia, Dalgalarrondo (2008) sobre depressão, Lipp (2001) e Homann et al (2012) para elucidar conceitos de estresse, todos fundantes para o processo de sistematização de informações e escrita deste trabalho. Para realizar a presente análise, partimos da metodologia de revisão bibliográfica integrativa, a partir da qual analisamos 4 artigos científicos coletados das plataformas digitais: Google Scholar, SciELO e PePsic. Para isto, partimos da seguinte pergunta norteadora: "qual a relação entre a fibromialgia e a depressão denotada nas pesquisas mais recentes?" Enquanto resultados, pudemos perceber a escassez de pesquisas recentes na área, principalmente na Psicologia, além de todas as pesquisas serem realizadas com o público feminino, por esse ser mais afetado pelo transtorno fibromiálgico. Além disso, é perceptível uma forma reducionista de lançar luz sobre a depressão, como se esta fosse apenas um sintoma da fibromialgia que pode ser desenvolvido ou não, negando o caráter individual da doença. Não se verificou uma abordagem sobre o ponto de vista psicopatológico e nem mesmo uma causa bem definida para o surgimento da doença em questão. A partir disso, pôde-se concluir a necessidade de uma maior investigação sobre os casos de fibromialgia e seu surgimento, qual a sua relação com as condições socioeconômicas, educacionais e de gênero. Além disso, é necessária uma fomentação nas pesquisas científicas acerca do tema.
\end{abstract}

Palavras-Chave: Depressão, Fibromialgia, Psicologia.

\section{RESUMEN}

El alcance de este artículo es analizar cómo se establece la relación entre el Síndrome de Fibromialgia y la depresión, teniendo en cuenta la gran demanda de pacientes afectados por las enfermedades. Para apoyarnos teóricamente, partimos de la concepción de Mello-Filho et al (2010) sobre la fibromialgia, Dalgalarrondo (2008) sobre la depresión, Lipp (2001) y Homann et al (2012) para dilucidar los conceptos de estrés, todos fundamentales para el proceso de sistematización de la información y redacción de este trabajo. Para realizar este análisis, partimos de la metodología integradora de revisión de literatura, a partir de la cual analizamos 4 artículos científicos recopilados de plataformas digitales: Google Scholar, SciELO y PePsic. Para esto, partimos de la siguiente pregunta orientadora: "¿cuál es la relación entre la fibromialgia y la depresión denotada en la investigación más reciente?" Como resultado, pudimos ver la escasez de investigación reciente en el área, especialmente en psicología,

\footnotetext{
${ }^{1}$ Graduanda em Psicologia, Centro Universitário Guararapes (UNIFG), karolinypaula.pt@gmail.com

${ }^{2}$ Técnico em Segurança do Trabalho, Instituto Federal de Pernambuco (IFPE), higorbezerralima2003@gmail.com

${ }^{3}$ Mestre em Gerontologia, Professor do Centro Universitário Guararapes (UNIFG), andre.cabral@unifg.com.br
} 
además de toda la investigación que se realiza. con el público femenino, ya que esto se ve más afectado por el trastorno de fibromialgia. Además, se nota una forma reduccionista de arrojar luz sobre la depresión, como si fuera solo un síntoma de fibromialgia que puede desarrollarse o no, negando el carácter individual de la enfermedad. No hubo un enfoque psicopatológico y ni siquiera una causa bien definida para el inicio de la enfermedad en cuestión. A partir de esto, se concluyó que existe la necesidad de una mayor investigación sobre los casos de fibromialgia y su aparición, cuál es su relación con las condiciones socioeconómicas, educativas y de género. Además, se requiere fomentar la investigación científica sobre el tema.

Palabras Clave: Depresión, Fibromialgia, Psicología.

\section{Abstract}

The scope of this article is to analyze how the relationship between Fibromyalgia Syndrome and depression occurs, given the high demand of patients affected by the diseases. To support ourselves theoretically, we start from the conception of Mello-Filho et al (2010) about fibromyalgia, Dalgalarrondo (2008) about depression, Lipp (2001) and Homann et al (2012) to elucidate concepts of stress, all key to the process. systematization of information and writing of the work. To perform this analysis, we started from the integrative literature review methodology, with which we analyzed 4 scientific articles collected from digital platforms: Google Scholar, SciELO and PePsic. For this, we start from the following guiding question: "What is the relationship between fibromyalgia and depression denoted in the most recent research?". As results, we could see the scarcity of recent research in the area, especially in Psychology, and all research is conducted with the female public, as this is more affected by fibromyalgia disorder. Moreover, a reductionist way of shedding light on depression is noticeable, as if it were just a symptom of fibromyalgia that may or may not develop, denying the individual character of the disease. There was no psychopathological approach and not even a well-defined cause for the onset of the disease in question. From this, it was concluded that there is a need for further investigation into the cases of fibromyalgia and its emergence, what is its relationship with socioeconomic, educational and gender conditions. In addition, a fostering of scientific research on the subject.

Keywords: Depression, Fibromyalgia, Psychology.

\section{INTRODUÇÃO}

O presente artigo busca verificar o que há na literatura especializada atual sobre a ocorrência da Fibromialgia (FM) em pacientes portadores de depressão. A FM é definida como "uma entidade clínica caracterizada por dor crônica com duração maior que três meses" e que acomete primordialmente mulheres (NUNES et al, 2012. p. 282). As quais, na sua maioria, quando afetadas pela patologia, possuem elevados níveis de estresse comparadas ao grupo sem fibromialgia (não FM). Isso porque, segundo os estudos de Calais, Andrade e Lipp (2003) e Sadir, Bignotto e Lipp (2010), as mulheres estão mais expostas à situação de estresse, tanto por sua condição biológica quanto pelos papéis culturais que a sociedade historicamente lhes impõe.

Logo, buscamos averiguar na literatura como a relação entre a psicopatologia e a FM é entendida, levando em consideração as visões de somatização. Adota-se para as e reflexões sobre a possibilidade de somatização a visão da relação entre mente-corpo e os mecanismos de 
produção de enfermidades, geralmente impulsionados por fenômenos do estresse (MELLOFILHO et al, 2010. p. 29). Isto é, há um deslocamento do sofrimento psíquico para um sintoma físico, no caso da pesquisa em questão, às dores corpóreas e o conjunto de sintomas relacionados a FM. Deslocamento pode ser definido, de acordo com Silva (2010), como um "mecanismo psicológico de defesa onde a pessoa substitui a finalidade inicial de uma pulsão por outra socialmente mais aceita" (p. 5), ou seja, é uma estratégia mental para canalização da dor, de acordo com a vertente psicanalítica.

A depressão "tem como elementos mais salientes o humor triste e o desânimo" (DEL PINO, 2003 apud DALGALARRONDO, 2008. p. 307). Entretanto, Dalgalarrondo (2008. p. 307-308) elucida a noção de que há uma "multiplicidade de sintomas afetivos, instintivos e neurovegetativos, ideativos e cognitivos, relativos à autovaloração, à vontade e à psicomotricidade", ou seja, os transtornos depressivos ultrapassam a noção de uma tristeza forte e persistente, afetando a saúde física e o bem-estar do portador. A persistência da dor física durante a vivência de uma dor psicológica descrita por relatos de caso ${ }^{4}$ fortalece a hipótese e apontamentos de que a FM é um transtorno psicossomático.

A pesquisa e reflexão acerca da FM e da sua correlação com as doenças psicológicas envolvidas no presente artigo é de extrema importância, visto a gravidade das doenças e como elas impactam no status quo vital dos portadores.

A dificuldade na identificação das causas do surgimento da FM e do seu tratamento acaba por marginalizar os portadores a partir do viés de saúde pública, e prejudicar o próprio sistema de saúde, afinal, sem o conhecimento prático acerca da doença e da influência das patologias psicológicas no seu surgimento e tratamento, não há como definir métodos de prevenção e tratamento, evidenciando assim, uma maior quantidade de consultas paliativas e de tempo útil utilizando medicamentos aliviadores que poderiam ser evitados com um tratamento mais direcionado (RAMIRO et al., 2013).

Portanto, visa-se a verificação literária especializada de como andam as pesquisas acerca da FM enquanto consequência das doenças psicológicas aqui investigadas. Logo, enquanto hipótese, pensamos que as psicopatologias, como a ansiedade e depressão, suscitam um processo de somatização da dor psíquica, motivando a sintomatologia da FM. Sendo assim, busca-se verificar se a depressão e a FM são associadas ou apenas coexistem.

Sendo assim, o método escolhido foi a Revisão de Literatura de base integrativa, uma vez que esse método dá margem a um maior cuidado com temas voltados para a área da saúde,

\footnotetext{
${ }^{4}$ NUNES et al. Transtorno depressivo e fibromialgia: associação com estresse de vida precoce. Relato de caso. Revista Dor. São Paulo, v. 13 n. 3 p. 282-286, jul./set., 2012.
} 
pois correlaciona dados teóricos e empíricos.

Enquanto objetivo geral, buscamos verificar se a depressão é uma patologia que suscita o aparecimento dos sintomas da FM e analisar o motivo dessa ocorrência, partindo do pressuposto da somatização dos sofrimentos psíquicos.

Enquanto objetivos específicos, há a necessidade de: investigar a FM enquanto doença psicossomática e a sua correlação com a psique; e, primordialmente, revisitar o olhar teórico descrito até agora sobre a influência das questões psicossomatopatológicas no surgimento da FM.

\section{FUNDAMENTAÇÃO TEÓRICA}

Visando elucidar conceitos essenciais para o desenvolvimento do artigo, como os de estresse, depressão e somatização, o presente artigo se debruça sobre os escritos de Dalgalarrondo (2008) e Mello-Filho \& Burd (2010), pois estes são autores fundamentais para os estudos das psicopatologias, sua sintomatologia e como estas condições afetam o indivíduo.

Geralmente, os dados achados no campo científico são voltados para as outras ciências da saúde, sempre em busca de novas formas de cura ou com vistas a explicar e diagnosticar a FM a partir puramente do viés biológico, o que é complexo, haja visto que os sintomas da FM são "desvinculados de alterações orgânicas passíveis de detecção e invariavelmente relacionadas a alterações no humor, na memória e no sono" (CLAUW, 2014; BELLATO et al., 2012 apud PELLOSI e PERES, 2017).

Em vista disso, a supervalorização das ciências biológicas e exatas em detrimento dos conhecimentos voltados para o entendimento do humano, ou seja, o positivismo científico, infelizmente ainda em voga no meio acadêmico, "tem levado muitos pesquisadores - inclusive no campo da Psicologia - a negligenciarem os aspectos subjetivos da fibromialgia" (PELLOSI e PERES, 2017). Ou seja, há uma necessidade de lançar luz sobre a FM a partir do olhar psicológico alinhado ao olhar biológico, para criar formas eficazes de identificar, diagnosticar e tratar os pacientes acometidos dessa síndrome.

Faz-se necessário ainda compreender as concepções encontradas na literatura sobre a condição do estresse, uma vez que este é frequentemente percebido como provável desencadeador da FM. Entende-se por estresse o conjunto de reações psicofisiológicas e comportamentais complexas, cuja origem está na necessidade de o organismo estabelecer a homeostase interna frente a uma situação ameaçadora (LIPP, 2001).

A desestabilização acontece quando o próprio organismo necessita responder a uma demanda que supere sua capacidade adaptativa. Esse processo, dependendo da quantidade extra 
de demanda, gera necessidade de adaptação do organismo e, como consequência, exerce papel fundamental na patogênese do estresse (LIPP, 2001). Logo, é perceptível então, que na síndrome complexa da FM, o conjunto de sintomas extrapola a capacidade adaptativa do ser (HOMANN et al, 2012).

Na atualidade, a partir de Lipp (2000), entende-se a evolução do estresse como um processo que compreende quatro fases, abordadas no Inventário de Sintomas de Estresse para adulto de Lipp (ISSL), são elas: a fase de alerta, fase de resistência, fase de quase-exaustão e a fase de exaustão. A primeira é entendida como o momento positivo do estresse, ou seja, um estresse mais natural para lidar com as atitudes adversas. A persistência desse estresse pode configurar a fase de resistência, que pode ser entendida como um esforço do organismo para se adaptar, que acaba num cansaço do mesmo. Quando o processo continua, entra-se na terceira fase, que é caracterizada por sintomas clínicos importantes que denotam o cansaço, mas ainda não é um esgotamento completo. Quando os fatores estressores continuam, é caracterizada uma fase de quase-exaustão, na qual se é observado que a reserva de energia do organismo se exaure, afetando o sistema imunológico como um todo (LIPP, 2000).

O estresse é um sistema amplo, não só com fases, mas também com tipos e formas de combate. A fase de exaustão (última fase do estresse) pode ser o estopim para outras como depressão e ansiedade, levando até mesmo o trabalhador ao interrupção laboral.

De acordo com Besset et al (2010) "o momento em que irrompe um mal-estar e a esse mal-estar é atribuído um nome, por exemplo, o nome de uma doença, o corpo desse sujeito fica, para sempre, afetado por um efeito de nomeação, marca do significante sobre sua carne” (p. 1258), ou seja, quando um sujeito é minimizado ao seu quadro fisiológico, em detrimento de sua subjetividade e seu ser psicológico, há uma marcação cujo sujeito nunca se livra completamente.

Os fatores estressores, a longo prazo, podem culminar em uma série de transtornos dentre eles o tratado na presente pesquisa, a depressão, a qual pode apresentar duas formas: os episódios e o transtorno recorrente. Um episódio depressivo pode ser: leve, moderado ou grave com ou sem sintomas psicóticos, a recorrência desses episódios é que caracteriza um transtorno (DATASUS, 2008). Classificado no CID 10 com o código F32 e F33, a depressão e suas formas são descritas enquanto um transtorno bem delimitado de humor, no qual

\footnotetext{
o paciente apresenta um rebaixamento do humor, redução da energia e diminuição da atividade [...] alteração da capacidade de experimentar o prazer, perda de interesse, diminuição da capacidade de concentração, associadas em geral à fadiga importante, mesmo após um esforço mínimo. (DATASUS, 2008).
}

A partir do aporte teórico acerca dos principais temas abordados, foi possível realizar 
uma pesquisa que poderá contribuir ainda que de maneira preliminar ou introdutória aos estudos sobre a temática desenvolvida.

\section{METODOLOGIA}

Enquanto metodologia de pesquisa, utilizou-se a Revisão de Literatura Integrativa, que consiste em um instrumento próprio da Prática Baseada em Evidências, a qual é constituída dos seguintes passos:

a definição do problema clínico, a identificação das informações necessárias, à condução da busca de estudos na literatura e sua avaliação crítica, a identificação da aplicabilidade dos dados oriundos das publicações e a determinação de sua utilização para o paciente (SOUZA et al, 2010. p. 102)

Dentre os modos de Revisão de Literatura disponíveis e amplamente utilizados, como a sistemática, a escolhida foi a Integrativa, pois, ao combinar dados da literatura teórica e empírica, abre-se um leque de propósitos (SOUZA et al, 2010.). Sendo assim, dialoga-se melhor com questões como o cuidado com o humano, ponto fundamental e basilar da psicologia. Logo, buscaremos analisar artigos em português e inglês que estejam na base de dados PePsic (Periódicos Eletrônicos em Psicologia), SciELO (Scientific Electronic Library Online) e no Google Scholar.

Enquanto critério de inclusão, utilizaremos em nosso recorte textos que versem acerca de depressão e FM, num recorte temporal dos últimos 10 anos. Os textos utilizados serão apenas artigos científicos, ou seja, os critérios de exclusão são: quaisquer textos que não sejam artigos científicos; que estejam em outra língua que não o português ou o inglês; textos que tenham como metodologia a revisão de literatura; ou textos que correspondam a todos os critérios, mas que não verse atentamente sobre o subtema de pesquisa do presente artigo.

Durante a pesquisa primeira, foram identificados 4 textos no PePsic, 4 no Scielo e 36 no Google Scholar. Após a triagem e enquadramento dos critérios acima citados, delimitamos 4 artigos que foram analisados na presente pesquisa. Para ilustrar melhor os dados analisados na pesquisa, segue abaixo um quadro com as devidas informações.

Para a realização das tarefas descritas anteriormente, partimos da seguinte pergunta norteadora: qual a relação entre a fibromialgia e a depressão denotada nas pesquisas mais recentes? 


\section{RESULTADOS E DISCUSSÃO}

Quadro 1: quadro descritivo dos artigos analisados.

\begin{tabular}{|c|c|c|c|}
\hline Procedência & Título do artigo & Autores & Periódico (vol, no, pág, ano) \\
\hline PEPSIC & $\begin{array}{l}\text { Neuropsychological assessment of } \\
\text { patients with fibromyalgia. }\end{array}$ & $\begin{array}{l}\text { GOULART, R.; } \\
\text { PESSOA, C. e } \\
\text { LOMBARDI } \\
\text { JUNIOR, I. }\end{array}$ & $\begin{array}{l}\text { Estud. psicol. (Natal) [online]. vol.22, } \\
\text { n.3, p. 264-273, } 2017 .\end{array}$ \\
\hline SCIELO & $\begin{array}{l}\text { Investigação do estresse, ansiedade e } \\
\text { depressão em mulheres com } \\
\text { fibromialgia: um estudo comparativo. }\end{array}$ & $\begin{array}{l}\text { RAMIRO, } \\
\text { Fernanda de } \\
\text { Souza et al. }\end{array}$ & $\begin{array}{l}\text { Rev. Bras. Reumatol. [online]. vol.54, } \\
\text { n.1, p. } 27-32,2014 .\end{array}$ \\
\hline $\begin{array}{l}\text { Google } \\
\text { Scholar }\end{array}$ & $\begin{array}{l}\text { A prática de caminhada diminui a } \\
\text { depressão em pacientes com síndrome } \\
\text { de fibromialgia. }\end{array}$ & $\begin{array}{l}\text { ANDRADE, } \\
\text { Alexandro et al }\end{array}$ & $\begin{array}{l}\text { ConScientiae Saúde, vol. 15, núm. 2, } \\
\text { 2016, pp. 217-224 }\end{array}$ \\
\hline $\begin{array}{l}\text { Google } \\
\text { Scholar }\end{array}$ & $\begin{array}{l}\text { Avaliação de Flexibilidade, Tender } \\
\text { Points e nível de ansiedade e depressão } \\
\text { em pacientes com síndrome da } \\
\text { fibromialgia. }\end{array}$ & $\begin{array}{l}\text { BARROS, T. R. } \\
\text { M. et al. }\end{array}$ & Colloq Vitae, vol. 7, nº 2, p. 13-19, 2015. \\
\hline
\end{tabular}

Fonte: própria.

Um ponto a ser destacado durante a construção do trabalho foi obtida durante a realização da pesquisa é escassez de artigos voltados ao viés psicológico da Fibromialgia denotando a necessidade de uma investigação mais ampla e profunda sobre o tema ainda tão pouco elucidado pela literatura e que acomete tantas pessoas. A necessidade de estudos mais aprofundados sobre as relações entre depressão e FM se torna ainda mais urgente quando nos deparamos com o significativo aumento de casos, já que no Brasil a quantidade de pessoas com FM chega na faixa de $3 \%$ da população, nos quais $80 \%$ das afetadas são mulheres na faixa etária de 35 à 55 anos (BRASIL, 2017). Dos quatro artigos analisados, 75\% são de áreas do conhecimento como a reumatologia, e apenas um é voltado pra a Psicologia. Quando há pouquíssimos estudos recentes desenvolvidos por profissionais da psicologia a área, o entendimento acerca da patologia, o seu diagnóstico, prognóstico e tratamento torna-se complexo para profissionais e pacientes.

Segundo Ramiro et al. (2013), que realizou uma pesquisa com uma amostragem de 50 mulheres, sendo 25 com FM e 25 com não FM, de idade de 20 a 65 anos, utilizando o Inventário de Depressão de Beck (BDI), um formulário que contém 21 afirmações que se referem a sintomas cognitivos e afetivos, sensações somáticas e desempenho, há um maior índice de depressão em pessoas com Fibromialgia. A pesquisa obteve como resultado uma média de 21,48 pontos para o grupo com FM. Um elevado nível médio comparado ao grupo com não FM, que obteve uma média de 7,36 pontos. Indicando assim, a presença de depressão moderada no grupo com FM, enquanto no não FM indica a ausência de depressão. 
Ainda assim, Barros et al. (2019) a partir de um estudo de caráter qualitativo comparativo dos resultados desses testes antes e após dois meses de intervenção por hidroterapia, exercícios aeróbios e alongamento no tratamento da FM, realizado com oito participantes do sexo feminino, com avaliação do questionário HADS - que possui um total de 14 itens, para a avaliação da depressão (HADS-D), número de tender points e teste terceiro dedo-chão, chega à conclusão de que houve diminuição no nível de depressão de pacientes que possuem FM, quando analisados antes e após a intervenção. Obtendo, então, a confirmação que hidroterapia e exercícios aeróbicos são importantes fatores que aliados aos tratamento psicológico e médico da FM, auxilia na diminuição dos reflexos negativos da FM, como a depressão, evidenciado pelo fato de além dos fatores biológicos, proporcionar a minimização do isolamento dessas pacientes e possíveis chances de estopim para ingresso em transtorno ou episódio depressivo.

Segmento esse ainda abordado por Andrade et al. (2016), que realizou um estudo clínico de delineamento pré e pós-tratamento, sem grupo controle, com 12 mulheres diagnosticadas com FM, com idade entre 18 a 70 anos. O objetivo desse estudo foi de verificar o efeito de sessões de caminhada, com duração de 60 minutos cada, e realização de 2 vezes na semana, com duração de 8 semanas, sobre a depressão em mulheres com fibromialgia. Como resultado verificou-se que o escore médio de depressão, que foi calculado a partir do BDI, diminuiu de 22,75 para 14,33. Evidenciando que existiu uma melhora, tendo em vista que ao início do programa de caminhada 41,6\% dos participantes apresentaram depressão grave e após a intervenção a maioria apresentou depressão mínima. Mas ainda assim, os mesmos autores afirmam que "é necessário definir um protocolo padrão que possa servir de guia na prática de atividades físicas para essa população".

E no estudo elaborado por Goulart et al (2017), foram avaliadas 32 mulheres com diagnóstico de FM, todas as voluntárias da pesquisa passaram por uma bateria de testes neuropsicológicos voltados para: memória, atenção, funções executivas, dor percebida, ansiedade e depressão. O objetivo do artigo era verificar as associações entre a sintomatologia da depressão, ansiedade, percepção de dor e déficits cognitivos em pacientes com FM. A conclusão foi que há uma coexistência da ansiedade e depressão nas pacientes, mas o quadro de ansiedade é mais relevante, ao passo que o nível de dor é mais alto e os prejuízos cognitivos mais evidentes, prejudicando significativamente a memória e atenção. Pacientes fibromiálgicos parecem ser menos eficazes ao desempenharem tarefas relacionadas às funções executivas.

\section{- Condições socioeconômicas e de gênero}

Pudemos constatar que, em todos os textos, o público de participantes era composto por 
mulheres, reforçando o fato de que, como dito anteriormente, a doença atinge majoritariamente a população feminina. A partir dos estudos de Andrade et al (2016), foi possível identificar que a maioria das mulheres do estudo tinham como agravante a condição socioeconômica e o nível de escolaridade mais baixo. Podemos considerar a relevância desses dados para o surgimento da depressão, afinal, a partir do estudo realizado por Santos e Kassouf (2007), constata-se que as condições socioeconômicas podem interferir na probabilidade de desenvolvimento dos sintomas depressivos, mas o efeito destas condições é superior nas mulheres. Além disso, ainda de acordo com Santos e Kassouf (2007), um maior nível educacional pode diminuir as chances do desenvolvimento desta patologia.

Então, se considerarmos a fibromialgia e a depressão enquanto comorbidades, podemos entender que os fatores sociais e econômicos impactam diretamente no surgimento e desenvolvimento das doenças, pois interferem na construção de subjetividade do indivíduo. No entanto, apesar da influência do fator econômico sobre a depressão, apenas o estudo acima citado denotou-a, ainda que de forma sucinta. Já a disparidade entre os gêneros afetados é informada em todos os estudos analisados.

Apenas no artigo desenvolvido por Ramiro et al (2014), há um parágrafo no qual se pondera o porquê de haver mais mulheres acometidas pelo mal da FM, explicitando o fato de que "estudos apontam que as mulheres estão mais expostas a situações de estresse, tanto pela sua condição biológica quanto pelos papéis culturais que a sociedade historicamente lhes impõe" (RAMIRO et al, 2014. p. 28). Dentre os autores estudados, Ramiro et al (2014) foram os únicos autores que se debruçaram em pontuar um possível porque de haver mais casos no público feminino, mesmo que não havendo uma explanação profunda sobre o tema.

Fica explícito a necessidade de pensar não só na FM ou na depressão, mas no recorte social que estas estão inseridas e como uma patologia impacta na outra, logo, fica claro a urgência de um maior quantitativo de pesquisas sobre o tema, principalmente do viés psicológico, considerando o indivíduo enquanto biopsicossocial e espiritual, como preconiza a OMS, visando esclarecer essa associação.

\section{- A visão partilhada de depressão}

Em todos os textos analisados os autores encaram a depressão como um sintoma que surge em decorrência da FM, que dificulta a vida dos pacientes acometidos pela patologia. Em Andrade et al (2016), a visão que se tem de depressão é como uma experiência que dificulta muito e a vida das pessoas fibromiálgicas e sua melhora. Ou seja, para esse autor, não há uma comorbidade, mas uma influência notória.

A mesma visão é sustentada por Barros et al (2015), que nãog explicita no artigo uma 
informação concreta acerca da depressão, apenas sinaliza a importância da análise emocional do paciente, evidenciando que "há prejuízos desde o desempenho profissional até o social, desestabilizando as relações familiares, restringindo o contato social e interferindo nos hábitos e rotinas dos pacientes, enfim, no geral há uma piora da qualidade de vida" (BARROS et al, 2015. p. 15).

Em Goulart, Pessoa e Júnior (2017), a literatura é mais voltada para a neurologia e a neuropsicologia e é evidenciado que "em dois estudos clássicos, há uma mudança no volume cerebral dos pacientes, mas em diferentes regiões" (p. 265). Ou seja, a FM causa danos biológicos ao cérebro, incluindo, ainda de acordo com o mesmo estudo, a diminuição na produção do neurotransmissor dopamina, que é responsável, dentre outras funções, pela sensação de prazer. Logo, é percebido que há uma relação, mesmo que indireta, com o surgimento dos sintomas da depressão.

A partir dos apontamentos anteriores, foi possível compreender que as pesquisas não denotar uma visão da depressão enquanto um transtorno bem definido, como ele é descrito no CID-10. A visão é apenas de uma consequência da FM, em decorrência da dor e das dificuldades enfrentadas pelos indivíduos acometidos. Não houve nenhum artigo que versou acerca do caráter psicopatológico que pode ser adotado enquanto ponto de vista, apenas Ramiro et al (2014) traz a visão do estresse como precursor da piora da patologia analisada.

\section{- A Síndrome da Fibromialgia em uma perspectiva de cura e tratamento.}

Todos os artigos trazem a visão de que a doença não tem uma causa definida, o que é condizente com a premissa trazida pela Sociedade Brasileira de Reumatologia, de que "alguns pacientes com FM desenvolvem a condição após um gatilho, como uma dor localizada maltratada, um trauma físico ou uma doença grave.” (SBR, 2019). Essa falta de informação pode estar associada à falta de pesquisas na área, como já foi indicado posteriormente, e a falta de socialização do conhecimento acadêmico, o que dificulta o diagnóstico da doença e, posteriormente, o seu prognóstico e um plano efetivo de tratamento para o sujeito.

Há uma consonância entre os textos de que "não existem cura ou tratamentos que sejam considerados muito eficazes" (BARROS et al, 2015. p. 14) apenas tratamentos e práticas que possam melhorar a qualidade de vida do paciente, como "o incremento da prática de atividade física (que) pode ser considerado como um tratamento importante em pessoas com síndrome de fibromialgia" (ANDRADE, 2014. p. 218).

Há menção a importância do trabalho da psicologia no quadro de um paciente fibromiálgico em apenas Barros et al (2015), que preconiza sobre a importância da terapia cognitivo-comportamental. Não foi encontrado nenhum trecho dos textos que versasse sobre a 
importância da psicoterapia para o paciente, mesmo havendo sempre associações acerca da depressão nas pacientes. Além disso, há a indicação de uma abordagem específica sem justificativa clara sobre o motivo dela ser importante.

\section{CONSIDERAÇÕES FINAIS}

Portanto, a partir da leitura dos artigos e análise por meio da metodologia supracitada, pudemos compreender a importância de estudos que tenham enquanto temática central a Fibromialgia, principalmente na área da Psicologia. Além disso, o entendimento acerca da relação entre a depressão e a FM merece lugar de destaque, afinal, a grande maioria dos casos relatados disserta acerca da coexistência dessas duas patologias.

Durante a análise, foi possível observar a relação entre o surgimento das doenças e os fatores sociais e econômicos, como gênero e nível de escolaridade, mas não foi identificada nenhuma relação com o local de residência dos participantes, não havendo de forma explícita um lugar onde há mais casos, por exemplo. A não consideração desta dimensão da realidade do público pode dificultar tanto o diagnóstico quanto o prognóstico de pacientes acometidas(os) pela doença.

Desse modo, há um consenso entre os autores acerca da depressão enquanto um sintoma que surge em decorrência da FM, e não como um transtorno bem delimitado na fundamentação teórica, a partir do CID- 10 (DATASUS, 2008) e de Dalgalarrondo (2008). Lançar luz para a depressão dessa forma acaba por negligenciar aspectos subjetivos do paciente, como sua história de vida e sua forma de olhar para sua existência, descartando informações que poderiam ser associadas ao tratamento do indivíduo, espelhando uma maior expectativa de melhora.

Portanto, é visivelmente necessária uma fomentação das discussões sobre o tema, e esse artigo busca ser um pontapé inicial para levar os leitores a reflexão acerca da importância de tratar de forma humana tantos indivíduos que sofrem. Para tal, faz-se necessário o entendimento do tema e a consideração dos fatores intrínsecos de cada ser, que é único e ao mesmo tempo está inserido num contexto social que deve ser validado e reconhecido.

\section{REFERÊNCIAS}

ANDRADE, Alexandro et al. A prática de caminhada diminui a depressão em pacientes com síndrome da fibromialgia. Conscientiae Saúde, Santa Catarina, v. 15, n. 2, p.217-224, maio 2016.

BESSET, V. L. et al. Um nome para a dor: fibromialgia. Revista Mal-Estar e Subjetividade. Fortaleza, v. 10 n. 4 p. 1245-1269, dez., 2010. 
BRASIL. MINISTÉRIO DA SAÚDE. Fibromialgia: os desafios de uma doença invisível. 2017. Elaborada por Ana Cláudia Felizola. Disponível em: <http://www.blog.saude.gov.br/b463z2>. Acesso em: 17 out. 2019.

CALAIS, Sandra Leal; ANDRADE, Lívia Márcia Batista de; LIPP, Marilda Emmanuel Novaes. Diferenças de sexo e escolaridade na manifestação de Stress em adultos jovens. Psicologia: Reflexão e Crítica, [s.1.], v. 16, n. 2, p.257-263, 2003. FapUNIFESP (SciELO). http://dx.doi.org/10.1590/s0102-79722003000200005.

DALGALARRONDO, P. Psicopatologia e semiologia dos transtornos mentais. $2^{\mathrm{a}}$ ed. Porto Alegre: Artmed, 2008.

DATASUS. F30-F39: Transtornos do humor [afetivos]. 2008. Disponível em: <http://www.datasus.gov.br/cid10/V2008/WebHelp/f30_f39.htm>. Acesso em: 18 out. 2019.

GOULART, Rubens; PESSOA, Cinthia; LOMBARDI JÚNIOR, Império. Neuropsychological assessment of patients with fibromyalgia. Estudos de Psicologia, Natal, v. 22, n. 3, p.264-273, jul./set. 2017.

HOMANN, Diogo et al . Percepção de estresse e sintomas depressivos: funcionalidade e impacto na qualidade de vida em mulheres com fibromialgia. Rev. Bras. Reumatol., São Paulo , v. 52, n. 3, p. 324-330, Jun., 2012 . Disponível em: <http://www.scielo.br/scielo .php?script=sci_arttext\&pid=S0482-50042012000300003\&lng=en\&nrm=iso >. acesso em 17 Out. 2019. http://dx.doi.org/10.1590/S0482-50042012000300003.

LIPP, M. E. N. Inventário de Sintomas de Estresse para Adultos. São Paulo: Casa do Psicólogo, 2000.

LIPP, M. E. N. (Org.). Pesquisas sobre stress no Brasil: Saúde, ocupações e grupos de risco. Campinas: Papirus, 2001

MELLO-FILHO, J.; BURD, M. Psicossomática Hoje. $2^{\text {a }}$ ed. São Paulo: Artmed, 2010.

BARROS, T. R. M. et al Avaliação de Flexibilidade, Tender Points e nível de Ansiedade e Depressão em Pacientes com Síndrome da Fibromialgia. Colloquium Vitae. ISSN: 1984-6436, v. 7, n. 2, p. 13-19, 27 abr. 2016.

NUNES, S. O. V. et al. Transtorno depressivo e fibromialgia: associação com estresse de vida precoce. Relato de caso. Revista Dor. São Paulo, v. 13, n. 3, p. 282-286, jul./set., 2012.

PELLOSI, E. F.; PERES, R. S. A fibromialgia sob a ótica psicanalítica: um breve panorama. Polêm!ca, [S.1.], v. 17, n. 1, p. 1-15, abr. 2017. Disponível em: <https://www.epublicacoes.uerj.br/index.php/polemica/article/view/28292/20339>. Acesso em: 17 abr. 2019.

RAMIRO, Fernanda de Souza et al . Investigação do estresse, ansiedade e depressão em mulheres com fibromialgia: um estudo comparativo. Rev. Bras. Reumatol., São Paulo, v. 54, n. 1, p. 27-32, Fev. 2014 . Disponível em: <http://www.scielo.br/scielo.php? script=sci_arttext\&pid=S0482-50042014000100027\&lng=en\&nrm=iso>. Acesso em: 15 Out. 2019. http://dx.doi.org/10.1016/j.rbr.2013.04.006. 
SADIR, Maria Angélica; BIGNOTTO, Márcia Maria; LIPP, Marilda Emmanuel Novaes. Stress e qualidade de vida: influência de algumas variáveis pessoais. Paidéia, Ribeirão Preto, v. 20, n. 45, p.73-81, abr. 2010. FapUNIFESP (SciELO). http://dx.doi.org/10.1590/s0103$863 \times 2010000100010$.

SANTOS, Marcelo Justus dos; KASSOUF, Ana Lúcia. Uma investigação dos determinantes socioeconômicos da depressão mental no Brasil com ênfase nos efeitos da educação. Econ. Apl., Ribeirão Preto, v. 11, n. 1, p.5-26, jan./mar. 2007. Trimestral. Disponível em: <http://www.scielo.br/scielo.php?script=sci_arttext\&pid=S1413-80502007000100001>.

Acesso em: 09 out. 2019.

SILVA, E. B. T. Mecanismos de defesa do Ego. Portal dos Psicólogos. Belo Horizonte, 15 jul. de 2011. Disponível em: <http://www.psicologia.pt/artigos/textos/TL0212.pdf>. Acesso em: 20 set. de 2019.

SOCIEDADE BRASILEIRA DE REUMATOLOGIA. . Fibromialgia. 2019. Disponível em: $<$ https://www.reumatologia.org.br/doencas-reumaticas/fibromialgia-e-doencas-articularesinflamatorias/>. Acesso em: 14 out. 2019.

SOUZA, M. T.; SILVA, M. D.; CARVALHO, R. Revisão integrativa: o que é e como fazer. Einstein, São Paulo, v. 8, n. 1, p. 102-106, mar., 2010. 